\title{
Extinction and persistence of a stochastic SICA epidemic model with standard incidence rate for HIV transmission
}

\author{
Xiaodong Wang ${ }^{1}$, Chunxia Wang ${ }^{1}$ and Kai Wang ${ }^{1 *}$
}

\author{
"Correspondence: \\ wangkaimath@sina.com \\ ${ }^{1}$ Department of Medical \\ Engineering and Technology, \\ Xinjiang Medical University, Urumqi, \\ Xinjiang, 830017, P.R. China
}

\begin{abstract}
In this paper, a stochastic SICA epidemic model with standard incidence rate for HIV transmission is proposed. The sufficient conditions of the extinction and persistence in mean for the disease are established. Numerical simulations show that random perturbations can suppress disease outbreaks and the risk of HIV transmission can be reduced by reducing the transmission coefficient of HIV while increasing the strength of the stochastic perturbation.
\end{abstract}

Keywords: SICA epidemic model; Extinction; Persistence in mean; Stochastic perturbation

\section{Introduction}

To the best of our knowledge, the human immunodeficiency virus (HIV) is a retrovirus that causes HIV infection and, over time, acquired immunodeficiency syndrome (AIDS). There is no cure or vaccine to AIDS. However, antiretroviral (ART) treatment improves health, prolongs life, and substantially reduces the risk of HIV transmission. As more people receive antiretroviral therapy, the number of new HIV infections worldwide is approximately 2.3 million, a 33 per cent decline in new infections compared to 2001 . At the same time, the number of AIDS deaths is also declining, with approximately 1.6 million AIDS deaths in 2012, down from 2.3 million in 2005 [1]. This goes to show that access to antiretroviral therapy has a huge impact on HIV prevention. Parvaiz et al. consider a fractional-order HIV epidemic model with the inclusion of prostitution in the population and its consequences on the disease transmission [2]. In [3], the authors consider a nonlinear fractional order epidemic model for HIV transmission and analyze by including an extra compartment, namely the exposed class, to the basic SIR epidemic model. They show through numerical simulations that the control measures effectively increase the quality

(c) The Author(s) 2021. This article is licensed under a Creative Commons Attribution 4.0 International License, which permits use, sharing, adaptation, distribution and reproduction in any medium or format, as long as you give appropriate credit to the original author(s) and the source, provide a link to the Creative Commons licence, and indicate if changes were made. The images or other third party material in this article are included in the article's Creative Commons licence, unless indicated otherwise in a credit line to the material. If material is not included in the article's Creative Commons licence and your intended use is not permitted by statutory regulation or exceeds the permitted use, you will need to obtain permission directly from the copyright holder. To view a copy of this licence, visit http://creativecommons.org/licenses/by/4.0/. 
of life and age limit of the HIV patients. The authors in [4] consider the following model:

$$
\left\{\begin{array}{l}
\frac{d S(t)}{d t}=\lambda-\mu S(t)-\frac{\beta S(t) I(t)}{N(t)}, \\
\frac{d I(t)}{d t}=\frac{\beta S(t) I(t)}{N(t)}-(\mu+\gamma+\rho) I(t)+\alpha A(t)+\omega C(t), \\
\frac{d C(t)}{d t}=\gamma I(t)-(\mu+\omega) C(t), \\
\frac{d A(t)}{d t}=\rho I(t)-(\alpha+\mu+d) A(t),
\end{array}\right.
$$

where the parameters are:

- $N(t)$ : The total population at time $t$;

- $S(t)$ : Susceptible individuals at time $t$;

- $I(t)$ : HIV-infected individuals with no clinical symptoms of AIDS at time $t$;

- $C(t)$ : HIV-infected individuals under ART treatment with a viral load remaining low at time $t$;

- $A(t)$ : HIV-infected individuals with AIDS clinical symptoms at time $t$;

- $\lambda$ : Recruitment rate;

- $\mu$ : Natural death rate;

- $\beta$ : HIV transmission rate;

- $\gamma$ : HIV treatment rate for $I$ individuals;

- $\rho$ : Default treatment rate for $I$ individuals;

- $\alpha$ : AIDS treatment rate;

- $\omega$ : Default treatment rate for $C$ individuals;

- $d$ : AIDS induced death rate.

They found that when $R_{0}<1$, the disease-free equilibrium of system (1.1) is asymptotically stable; when $R_{0}>1$, the disease-free equilibrium is unstable and there is a globally asymptotically stable endemic equilibrium. Here, $R_{0}=\frac{\beta(\alpha+\mu+d)(\mu+\omega)}{\mu[(\mu+\omega)(\rho+\alpha+\mu+d)+\gamma(\alpha+\mu+d)+\rho d]+\rho \omega d}$ is the basic reproduction number.

The above studies did not consider the effect of white noise in the environment on the model; in fact, infectious diseases are inevitably affected by random white noise in the environment. May [5] finds that because of the fluctuation of the environment, the parameters of the deterministic system, such as the death rate and the transmission coefficient, and other parameters of the deterministic system show a certain degree of random fluctuation. Therefore, the transmission coefficient may be affected by many environmental factors, such as temperature, wind, rain, and snow. In [6], the authors extend the classical SIS epidemic model from a deterministic framework to a stochastic one and formulate it as a stochastic differential equation (SDE) for the number of infectious individuals $I(t)$. They discuss perturbation by stochastic noise. In the case of persistence they show the existence of a stationary distribution and derive expressions for its mean and variance. In [7], the authors present the threshold of a stochastic SIQS epidemic model which determines the extinction and persistence of the disease and find that noise can suppress the disease outbreak. Therefore, when establishing the corresponding mathematical model, we must consider the impact of white noise on the disease. Many scholars have introduced white noise into the infectious disease model [8-12]. In addition, there are a number of other types of stochastic models that have been developed to further explain that stochastic factors are integral to the modeling of infectious diseases, see [13-16].

In this paper, our aim is to introduce random white noise in the environment into the deterministic model and to study the effect of random disturbance on the number of HIV 
infected people and the conditions between the random disturbance and the parameters of the model, and if the number of HIV infected people can be controlled. Motivated by [6], we consider here random white noise in the environment, which is assumed to demonstrate itself as fluctuations in the parameter $\beta$, so that $\beta \rightarrow \beta+\sigma d B(t)$, where $B(t)$ is a standard Brownian motion with intensity $\sigma^{2}>0$. Hence, we can derive the following stochastic model:

$$
\left\{\begin{array}{l}
d S(t)=\left[\lambda-\mu S(t)-\frac{\beta S(t) I(t)}{N(t)}\right] d t-\frac{\sigma S(t) I(t)}{N(t)} d B(t), \\
d I(t)=\left[\frac{\beta S(t) I(t)}{N(t)}-(\mu+\gamma+\rho) I(t)+\alpha A(t)+\omega C(t)\right] d t+\frac{\sigma S(t) I(t)}{N(t)} d B(t), \\
d C(t)=[\gamma I(t)-(\mu+\omega) C(t)] d t \\
d A(t)=[\rho I(t)-(\alpha+\mu+d) A(t)] d t
\end{array}\right.
$$

This paper is organized as follows. In Sect. 2, we prove that there is a unique global positive solution for system (1.2). In Sect. 3, we show that the disease goes to extinction exponentially under certain conditions and the persistence of the disease, that is to say, the disease will prevail. In Sect. 4, we carry out the numerical simulations to demonstrate the analytical results. In Sect. 5, we give some conclusions.

Throughout this paper, we let $\left(\Omega, \mathcal{F},\{\mathcal{F}\}_{t \geq 0}, \mathbb{P}\right)$ be a complete probability space with filtration $\{\mathcal{F}\}_{t \geq 0}$ satisfying the usual conditions (that is to say, it is increasing and right continuous while $\mathcal{F}_{0}$ contains all $\mathbb{P}$-null sets). On the other hand, we define $\mathbb{R}_{+}^{d}=\left\{x \in \mathbb{R}^{d}\left|x_{i}\right\rangle\right.$ 0 for all $1 \leq i \leq d\}$.

Generally speaking, consider the $d$-dimensional stochastic differential equation

$$
d x(t)=f(x(t), t) d t+g(x(t), t) d B_{t},
$$

where $f(t, x(t))$ is a function in $\mathbb{R}^{d}$ defined in $\left[t_{0}, \infty\right] \times \mathbb{R}^{d}$, and $g(x(t), t)$ is a $d \times m$ matrix, $f, g$ are locally Lipschitz functions in $x$. $B_{t}$ denotes an m-dimensional standard Brownian motion defined on the complete probability space $\left(\Omega, \mathcal{F},\{\mathcal{F}\}_{t \geq 0}, \mathbb{P}\right)$. Denote by $C^{2,1}\left(\mathbb{R}^{d} \times\right.$ $\left.\left[t_{0}, \infty\right] ; \mathbb{R}_{+}\right)$the family of all nonnegative functions $V(x(t), t)$ defined on $\mathbb{R}^{d} \times\left[t_{0}, \infty\right]$ such that they are continuously twice differentiable in $x$ and once in $t$. We define the differential operator $L$ of equation (1.3) by [17]

$$
L=\frac{\partial}{\partial t}+\sum_{i=1}^{d} f_{i}(x, t) \frac{\partial}{\partial x_{i}}+\frac{1}{2} \sum_{i, j=1}^{d}\left[g^{T}(x, t) g(x, t)\right]_{i j} \frac{\partial^{2}}{\partial x_{i} \partial x_{j}} .
$$

If $L$ acts on a function $V \in C^{2,1}\left(\mathbb{R}^{d} \times\left[t_{0}, \infty\right], \mathbb{R}_{+}\right)$, then

$$
L V(x, t)=V_{t}(x, t)+V_{x}(x, t) f(x, t)+\frac{1}{2} \operatorname{trace}\left[g^{T}(x, t) V_{x x}(x, t) g(x, t)\right],
$$

where $V_{t}(x, t)=\frac{\partial V}{\partial t}, V_{x}(x, t)=\left(\frac{\partial V}{\partial x_{i}}, \ldots, \frac{\partial V}{\partial x_{d}}\right), V_{x x}(x, t)=\left(\frac{\partial^{2} V}{\partial x_{i} \partial x_{j}}\right) d \times d$.

From Itô's formula, if $x(t) \in \mathbb{R}^{d}$, then

$$
d V(x, t)=L V(x, t) d t+V_{x}(x, t) g(x, t) d B_{t} .
$$




\section{Existence and uniqueness of positive solution}

Theorem 2.1 There is a unique solution $(S(t), I(t), C(t), A(t))$ of system (1.2) on $t \geq 0$ for any initial value $(S(0), I(0), C(0), A(0)) \in \mathbb{R}_{+}^{4}$, and the solution will remain in $\mathbb{R}_{+}^{4}$ with probability one, namely $(S(t), I(t), C(t), A(t)) \in \mathbb{R}_{+}^{4}$ for all $t \geq 0$ almost surely. Moreover,

$$
\frac{\lambda}{(\mu+d)}<\lim _{t \rightarrow \infty} N(t)=\frac{\lambda}{\mu}
$$

where $N(t)=S(t)+I(t)+C(t)+A(t)$.

Proof We can easily know that the coefficients of system (1.2) are locally Lipschitz continuous, then for any given initial value $(S(0), I(0), C(0), A(0)) \in \mathbb{R}_{+}^{4}$, there is a unique local solution $(S(t), I(t), C(t), A(t))$ on $t \in\left[0, \tau_{e}\right)$, where $\tau_{e}$ is the explosion time (see [17]). To show that this solution is global, we only need to prove that $\tau_{e}=\infty$ almost surely. Let $k_{0} \geq 0$ be sufficiently large so that $(S(0), I(0), C(0), A(0))$ all lie within the interval $\left[\frac{1}{k_{0}}, k_{0}\right]$. For each integer $k \geq k_{0}$, define the following stopping time:

$$
\begin{aligned}
\tau_{k}= & \inf \left\{t \in\left[0, \tau_{e}\right): \min \{(S(t), I(t), C(t), A(t))\} \leq \frac{1}{k}\right. \\
& \text { or } \max \{(S(t), I(t), C(t), A(t))\} \geq k\},
\end{aligned}
$$

where throughout this paper, we set $\inf \emptyset=\infty$ (as usual $\emptyset$ denotes the empty set). According to the definition of the stopping time, $\tau_{k}$ is increasing as $k \rightarrow \infty$. Set $\tau_{\infty}=\lim _{k \rightarrow \infty} \tau_{k}$, whence $\tau_{\infty} \leq \tau_{e}$ almost surely. Namely, we need to show that $\tau_{\infty}=\infty$ almost surely. We assumed that there exists a pair of constants $T>0$ and $\epsilon \in(0,1)$ such that

$$
P\left\{\tau_{\infty} \leq T\right\}>\epsilon .
$$

As a result, there is an integer $k_{1} \geq k_{0}$ such that

$$
P\left\{\tau_{k} \leq T\right\}>\epsilon \text { for all } k \geq k_{1} .
$$

Now define a $C^{2}$-function $V: \mathbb{R}_{+}^{4} \rightarrow \mathbb{R}_{+}$by

$$
V(t)=(S(t)-1-\ln S(t))+(I(t)-1-\ln I(t))+(C(t)-1-\ln C(t))+(A(t)-1-\ln A(t)) .
$$

Applying Itô's formula, we obtain

$$
d V(t)=L V(t) d t-\left[\frac{\sigma(S(t)-1) I(t)}{N(t)}-\frac{\sigma(I(t)-1) S(t)}{N(t)}\right] d B(t)
$$

where

$$
\begin{aligned}
L V(t)= & \left(1-\frac{1}{S(t)}\right)\left(\lambda-\mu S(t)-\frac{\beta S(t) I(t)}{N(t)}\right)+\frac{1}{2 S^{2}(t)} \frac{\sigma^{2} S^{2}(t) I^{2}(t)}{N^{2}(t)} \\
& +\left(1-\frac{1}{I(t)}\right)\left(\frac{\beta S(t) I(t)}{N(t)}-(\mu+\gamma+\rho) I(t)+\alpha A(t)+\omega C(t)\right)
\end{aligned}
$$




$$
\begin{aligned}
& +\frac{1}{2 I^{2}(t)} \frac{\sigma^{2} S^{2}(t) I^{2}(t)}{N^{2}(t)}+\left(1-\frac{1}{C(t)}\right)[\gamma I(t)-(\mu+\omega) C(t)] \\
& +\left(1-\frac{1}{A(t)}\right)[\rho I(t)-(\alpha+\mu+d) A(t)] \\
= & \lambda+4 \mu+\gamma+\rho+\alpha+\omega+d+\frac{\beta I(t)}{N(t)}+\frac{\sigma^{2} I^{2}(t)}{2 N^{2}(t)}+\frac{\sigma^{2} S^{2}(t)}{2 N^{2}(t)}-\mu N(t)-d A(t) \\
& -\frac{\lambda}{S(t)}-\frac{\beta S(t)}{N(t)}-\frac{\alpha A(t)+\omega C(t)}{I(t)}-\frac{\gamma I(t)}{C(t)}-\frac{\rho I(t)}{A(t)} \\
\leq & \lambda+4 \mu+\gamma+\rho+\alpha+\omega+d+\beta+\sigma^{2} \doteq K .
\end{aligned}
$$

Thus

$$
d V(t)=K d t-\left[\frac{\sigma(S(t)-1) I(t)}{N(t)}-\frac{\sigma(I(t)-1) S(t)}{N(t)}\right] d B(t) .
$$

Integrating both sides of (2.3) from 0 to $T \wedge \tau_{k}$ and taking expectations, we can obtain

$$
\begin{aligned}
& \mathbb{E} V\left(S\left(T \wedge \tau_{k}\right), I\left(T \wedge \tau_{k}\right), C\left(T \wedge \tau_{k}\right), A\left(T \wedge \tau_{k}\right)\right) \\
& \quad \leq V(S(0), I(0), C(0), A(0))+K T<\infty .
\end{aligned}
$$

Set $\Omega_{k}=\left\{\tau_{k} \leq t\right\}$ for $k \geq k_{1}$ by (2.2), $P\left(\Omega_{k}\right) \geq \epsilon$. Notice that, for every $\omega \in \Omega_{k}$, there is at least one of $\left(S\left(\tau_{k}, \omega\right), I\left(\tau_{k}, \omega\right), C\left(\tau_{k}, \omega\right), A\left(\tau_{k}, \omega\right)\right)$ that equals to $k$ or $\frac{1}{k}$. Consequently,

$$
V\left(S\left(\tau_{k}, \omega\right), I\left(\tau_{k}, \omega\right), C\left(\tau_{k}, \omega\right), A\left(\tau_{k}, \omega\right)\right) \geq(k-1-\log k) \wedge\left(\frac{1}{k}-1+\log k\right),
$$

where $a \wedge b$ denotes the minimum of $a$ and $b$. In view of (2.4) and (2.5), we have

$$
\begin{aligned}
V(S(0), I(0), C(0), A(0))+K T & \geq \mathbb{E}\left[1_{\Omega_{k}} V\left(S\left(\tau_{k}, \omega\right), I\left(\tau_{k}, \omega\right), C\left(\tau_{k}, \omega\right), A\left(\tau_{k}, \omega\right)\right)\right] \\
& \geq \epsilon\left[(k-1-\log k) \wedge\left(\frac{1}{k}-1+\log k\right)\right],
\end{aligned}
$$

where $1_{\Omega_{k}}$ is the indicator function of $\Omega_{k}$. Let $k \rightarrow \infty$ lead to the contradiction

$$
\infty>V(S(0), I(0), C(0), A(0))+K T=\infty .
$$

Therefore, we must have $\tau_{\infty}=\infty$ almost surely.

In view of system (1.2), we have

$$
\frac{d N(t)}{d t}=\lambda-\mu N(t)-d A(t)
$$

Solving this equation, we obtain that

$$
N(t)=\frac{\lambda}{\mu}+\left(N(0)-\frac{\lambda}{\mu}\right) e^{-\mu t}-d \int_{0}^{t} A(s) e^{-\mu(t-s)} d s,
$$


which implies that

$$
\lim _{t \rightarrow \infty} N(t)=\frac{\lambda}{\mu} \quad \text { almost surely. }
$$

On the other hand, we have

$$
\frac{d N(t)}{d t}>\lambda-(\mu+d) N(t)
$$

Then we can obtain

$$
N(t)>\frac{\lambda}{\mu+d}+e^{-(\mu+d) t}\left(N(0)-\frac{\lambda}{\mu+d}\right)
$$

which implies that

$$
\lim _{t \rightarrow \infty} N(t)>\frac{\lambda}{\mu+d} \quad \text { almost surely. }
$$

The proof of Theorem 2.1 is complete.

\section{Extinction and persistence in mean}

In this section, we discuss under what conditions the disease will be extinct and the persistence of the disease, namely, under what condition the disease will prevail. For convenience, firstly, we define $\langle X(t)\rangle=\frac{1}{t} \int_{0}^{t} X(s) d s$.

Theorem 3.1 If $R_{1}<1$ or $\sigma^{2} \leq \beta$ and $R_{2}<1$ hold, then the disease $I(t)$ will die out exponentially with probability one, that is,

$$
I(t), C(t), A(t) \rightarrow 0 \quad \text { as } t \rightarrow+\infty \quad \text { and } \quad S(t) \rightarrow \frac{\lambda}{\mu} \quad \text { as } t \rightarrow+\infty
$$

where

$$
R_{1}=\frac{\beta^{2}}{2 \sigma^{2} \mu}, \quad R_{2}=\frac{\beta}{\left(\mu+\frac{\sigma^{2}}{2}\right)} .
$$

Proof Let $Q(t)=I(t)+C(t)+A(t)$. Making use of Itô's formula, we can have

$$
\begin{aligned}
d \ln Q(t)= & \frac{1}{I(t)+C(t)+A(t)}\left[\frac{\beta S(t) I(t)}{N(t)}-\mu I(t)-\mu C(t)-(\mu+d) A(t)\right] d t \\
& -\frac{\sigma^{2} S^{2}(t) I^{2}(t)}{2 N^{2}(I(t)+C(t)+A(t))^{2}} d t+\frac{\sigma S(t) I(t)}{N(t)(I(t)+C(t)+A(t))} d B(t) \\
\leq & {\left[\frac{\beta S(t) I(t)}{N(t)(I(t)+C(t)+A(t))}-\mu-\frac{\sigma^{2} S^{2}(t) I^{2}(t)}{2 N^{2}(t)(I(t)+C(t)+A(t))^{2}}\right] d t } \\
& +\frac{\sigma S(t) I(t)}{N(t)(I(t)+C(t)+A(t))} d B(t) \\
= & {\left[-\left(\frac{\sigma S(t) I(t)}{\sqrt{2} N(t)(I(t)+C(t)+A(t))}-\frac{\beta \sqrt{2}}{2 \sigma}\right)^{2}+\frac{\beta^{2}}{2 \sigma^{2}}-\mu\right] d t }
\end{aligned}
$$




$$
\begin{aligned}
& +\frac{\sigma S(t) I(t)}{N(t)(I(t)+C(t)+A(t))} d B(t) \\
\leq & {\left[\frac{\beta^{2}}{2 \sigma^{2}}-\mu\right] d t+\frac{\sigma S(t) I(t)}{N(t)(I(t)+C(t)+A(t))} d B(t) } \\
= & {\left[\mu\left(R_{1}-1\right)\right] d t+\frac{\sigma S(t) I(t)}{N(t)(I(t)+C(t)+A(t))} d B(t) . }
\end{aligned}
$$

Integrating on both sides of equation (3.1) from 0 to $t$, and then dividing by $t$, we can obtain

$$
\frac{\ln Q(t)}{t} \leq \frac{\ln Q(0)}{t}+\mu\left(R_{1}-1\right)+\frac{M(t)}{t},
$$

where

$$
M(t)=\int_{0}^{t} \frac{\sigma S(s) I(s)}{N(s)(I(s)+C(s)+A(s))} d B(s) .
$$

By the large number theorem for martingale (see [17]), we can get

$$
\lim _{t \rightarrow+\infty} \frac{M(t)}{t}=0, \quad \text { almost surely. }
$$

In the light of (3.2) and (3.3), if $R_{1}<1$, then

$$
\limsup _{t \rightarrow+\infty} \frac{\ln Q(t)}{t} \leq \mu\left(R_{1}-1\right)<0, \quad \text { almost surely, }
$$

which implies that

$$
\lim _{t \rightarrow+\infty} I(t)=0, \quad \lim _{t \rightarrow+\infty} C(t)=0, \quad \lim _{t \rightarrow+\infty} A(t)=0, \quad \text { almost surely. }
$$

On the other hand, we consider the function $f(x)=\beta x-\frac{\sigma^{2} x^{2}}{2}$, where $x \in(0,1]$. One can obtain that if $\frac{\sigma}{\sqrt{2}} \leq \frac{\beta \sqrt{2}}{2 \sigma}$, that is, $\sigma^{2} \leq \beta, f(x)$ has the max value $f(1)=\beta-\frac{\sigma^{2}}{2}$. Let $x=$ $\frac{S(t) I(t)}{N(t)(I(t)+C(t)+A(t))}$, we have

$$
f(x)=-\left(\frac{\sigma S(t) I(t)}{\sqrt{2} N(t)(I(t)+C(t)+A(t))}-\frac{\beta \sqrt{2}}{2 \sigma}\right)^{2}+\frac{\beta^{2}}{2 \sigma^{2}} \leq \beta-\frac{\sigma^{2}}{2} .
$$

Therefore,

$$
\begin{aligned}
d \ln Q(t)= & \frac{1}{I(t)+C(t)+A(t)}\left[\frac{\beta S(t) I(t)}{N(t)}-\mu I(t)-\mu C(t)-(\mu+d) A(t)\right] d t \\
& -\frac{\sigma^{2} S^{2}(t) I^{2}(t)}{2 N^{2}(I(t)+C(t)+A(t))^{2}} d t+\frac{\sigma S(t) I(t)}{N(t)(I(t)+C(t)+A(t))} d B(t) \\
\leq & {\left[\frac{\beta S(t) I(t)}{N(t)(I(t)+C(t)+A(t))}-\mu-\frac{\sigma^{2} S^{2}(t) I^{2}(t)}{2 N^{2}(t)(I(t)+C(t)+A(t))^{2}}\right] d t } \\
& +\frac{\sigma S(t) I(t)}{N(t)(I(t)+C(t)+A(t))} d B(t) \\
= & {\left[-\left(\frac{\sigma S(t) I(t)}{\sqrt{2} N(t)(I(t)+C(t)+A(t))}-\frac{\beta \sqrt{2}}{2 \sigma}\right)^{2}+\frac{\beta^{2}}{2 \sigma^{2}}-\mu\right] d t }
\end{aligned}
$$




$$
\begin{aligned}
& +\frac{\sigma S(t) I(t)}{N(t)(I(t)+C(t)+A(t))} d B(t) \\
\leq & {\left[\beta-\frac{\sigma^{2}}{2}-\mu\right] d t+\frac{\sigma S(t) I(t)}{N(t)(I(t)+C(t)+A(t))} d B(t) } \\
= & {\left[\left(\mu+\frac{\sigma^{2}}{2}\right)\left(R_{2}-1\right)\right] d t+\frac{\sigma S(t) I(t)}{N(t)(I(t)+C(t)+A(t))} d B(t) . }
\end{aligned}
$$

Hence, if $R_{2}<1$, we obtain

$$
\limsup _{t \rightarrow+\infty} \frac{\ln Q(t)}{t} \leq\left(\mu+\frac{\sigma^{2}}{2}\right)\left(R_{2}-1\right)<0, \quad \text { almost surely, }
$$

which implies that

$$
\lim _{t \rightarrow+\infty} I(t)=0, \quad \lim _{t \rightarrow+\infty} C(t)=0, \quad \lim _{t \rightarrow+\infty} A(t)=0 \quad \text { almost surely. }
$$

Based on the above analysis, in view of (2.1), if $R_{1}<1$ or $\sigma^{2} \leq \beta$ and $R_{2}<1$, we have

$$
\lim _{t \rightarrow+\infty} S(t)=\frac{\lambda}{\mu} \quad \text { almost surely. }
$$

The proof is completed.

Theorem 3.2 For any initial value $(S(0), I(0), C(0), A(0)) \in \mathbb{R}_{+}^{4}$, if $R_{3}>1$, the disease is persistence in mean. Furthermore,

$$
\begin{aligned}
\liminf _{t \rightarrow+\infty}\langle I(t)\rangle & \geq \frac{\lambda(\omega+\mu)(\alpha+\mu+d)\left(\mu+\gamma+\rho+\frac{\sigma^{2}}{2}\right)}{\beta(\mu+d)[(\omega+\mu)(\alpha+\mu+d+\rho)+\gamma(\alpha+\mu+d)]}\left(R_{3}-1\right) \\
& >0 \quad \text { almost surely, }
\end{aligned}
$$

where

$$
R_{3}=\frac{\beta}{\mu+\gamma+\rho+\frac{\sigma^{2}}{2}}
$$

Proof Integrating system (1.2) from 0 to $t$, we can obtain

$$
\begin{aligned}
& \frac{S(t)-S(0)}{t}+\frac{I(t)-I(0)}{t}+\frac{\alpha}{\mu+\alpha+d} \frac{A(t)-A(0)}{t}+\frac{\omega}{\omega+\mu} \frac{C(t)-C(0)}{t} \\
& =\lambda-\mu\langle S(t)\rangle+\left[\frac{\alpha \rho}{\mu+\alpha+d}+\frac{\omega \gamma}{\omega+\mu}-(\mu+\gamma+\rho)\right]\langle I(t)\rangle .
\end{aligned}
$$

Then

$$
\langle S(t)\rangle=\frac{\lambda}{\mu}+\frac{1}{\mu}\left[\frac{\alpha \rho}{\mu+\alpha+d}+\frac{\omega \gamma}{\omega+\mu}-(\mu+\gamma+\rho)\right]\langle I(t)\rangle-\frac{H(t)}{\mu},
$$

where

$$
H(t)=\frac{S(t)-S(0)}{t}+\frac{I(t)-I(0)}{t}+\frac{\alpha}{\mu+\alpha+d} \frac{A(t)-A(0)}{t}+\frac{\omega}{\omega+\mu} \frac{C(t)-C(0)}{t} .
$$


In addition,

$$
\frac{C(t)-C(0)}{t}=\gamma\langle I(t)\rangle-(\omega+\mu)\langle C(t)\rangle,
$$

then

$$
\langle C(t)\rangle=-\frac{C(t)-C(0)}{(\omega+\mu) t}+\frac{\gamma}{\omega+\mu}\langle I(t)\rangle .
$$

Furthermore,

$$
\frac{A(t)-A(0)}{t}=\rho\langle I(t)\rangle-(\alpha+\mu+d)\langle A(t)\rangle,
$$

then

$$
\langle A(t)\rangle=-\frac{A(t)-A(0)}{(\alpha+\mu+d) t}+\frac{\rho}{\alpha+\mu+d}\langle I(t)\rangle .
$$

Define

$$
V(t)=-\ln I(t)+e,
$$

where $e$ is a constant. $e=-\min \{-\ln I(t)\}$ to keep the nonnegativity of $V(t)$. Applying Itô's formula, we obtain

$$
\begin{aligned}
d(V(t))= & -\frac{1}{I(t)}\left[\frac{\beta S(t) I(t)}{N(t)}-(\mu+\gamma+\rho) I(t)+\alpha A(t)+\omega C(t)\right] d t \\
& -\frac{\sigma S(t)}{N(t)} d B(t)+\frac{\sigma^{2} S^{2}(t)}{2 N^{2}(t)} d t \\
= & {\left[-\frac{\beta S(t)}{N(t)}+(\mu+\gamma+\rho)-\frac{\alpha A(t)+\omega C(t)}{I(t)}+\frac{\sigma^{2} S^{2}(t)}{2 N^{2}(t)}\right] d t-\frac{\sigma S(t)}{N(t)} d B(t) } \\
\leq & {\left[-\frac{\beta(N(t)-I(t)-C(t)-A(t))}{N(t)}+\mu+\gamma+\rho+\frac{\sigma^{2}}{2}\right] d t-\frac{\sigma S(t)}{N(t)} d B(t) } \\
= & {\left[-\beta+\mu+\gamma+\rho+\frac{\sigma^{2}}{2}+\frac{\beta I(t)}{N(t)}+\frac{\beta C(t)}{N(t)}+\frac{\beta A(t)}{N(t)}\right] d t-\frac{\sigma(t)}{N(t)} d B(t) . }
\end{aligned}
$$

According to $N(t)>\frac{\lambda}{\mu+d}$, we have

$$
\begin{aligned}
d(V(t)) \leq & {\left[-\beta+\mu+\gamma+\rho+\frac{\sigma^{2}}{2}+\frac{\beta I(t)}{N(t)}+\frac{\beta C(t)}{N(t)}+\frac{\beta A(t)}{N(t)}\right] d t-\frac{\sigma S(t)}{N(t)} d B(t) } \\
\leq & {\left[-\beta+\mu+\gamma+\rho+\frac{\sigma^{2}}{2}+\frac{\beta(\mu+d)}{\lambda}(I(t)+C(t)+A(t))\right] d t } \\
& -\frac{\sigma S(t)}{N(t)} d B(t) .
\end{aligned}
$$


Hence

$$
\begin{aligned}
\frac{\ln I(0)}{t}-\frac{\ln I(t)}{t} \leq & -\beta+\mu+\gamma+\rho+\frac{\sigma^{2}}{2}+\frac{\beta(\mu+d)}{\lambda}(\langle I(t)\rangle+\langle C(t)\rangle+\langle A(t)\rangle) \\
& -\frac{1}{t} \int_{0}^{t} \frac{\sigma S(s)}{N(s)} d B(s) .
\end{aligned}
$$

In the light of (3.6) and (3.7), we have

$$
\begin{aligned}
\langle I(t)\rangle \geq & \frac{\lambda}{\beta(\mu+d)}\left[\beta-\left(\mu+\gamma+\rho+\frac{\sigma^{2}}{2}\right)+\frac{\ln I(0)}{t}-\frac{\ln I(t)}{t}+\frac{1}{t} \int_{0}^{t} \frac{\sigma S(s)}{N(s)} d B(s)\right] \\
& -\langle C(t)\rangle-\langle A(t)\rangle \\
= & \frac{\lambda}{\beta(\mu+d)}\left[\beta-\left(\mu+\gamma+\rho+\frac{\sigma^{2}}{2}\right)+\frac{\ln I(0)}{t}-\frac{\ln I(t)}{t}+\frac{1}{t} \int_{0}^{t} \frac{\sigma S(s)}{N(s)} d B(s)\right] \\
& +\frac{C(t)-C(0)}{(\omega+\mu) t}-\frac{\gamma}{\omega+\mu}\langle I(t)\rangle+\frac{A(t)-A(0)}{(\alpha+\mu+d) t}-\frac{\rho}{\alpha+\mu+d}\langle I(t)\rangle .
\end{aligned}
$$

Furthermore,

$$
\langle I(t)\rangle \geq \frac{\lambda(\omega+\mu)(\alpha+\mu+d)\left(\mu+\gamma+\rho+\frac{\sigma^{2}}{2}\right)}{\beta(\mu+d)[(\omega+\mu)(\alpha+\mu+d+\rho)+\gamma(\alpha+\mu+d)]}\left(R_{3}-1\right)+F(t),
$$

where

$$
\begin{aligned}
F(t)= & \frac{(\omega+\mu)(\alpha+\mu+d)}{(\omega+\mu)(\alpha+\mu+d+\rho)+\gamma(\alpha+\mu+d)} \\
& \times\left\{\frac { \lambda } { \beta ( \mu + d ) } \left(\frac{\ln I(0)}{t}-\frac{\ln I(t)}{t}\right.\right. \\
& \left.\left.+\frac{1}{t} \int_{0}^{t} \frac{\sigma S(s)}{N(s)} d B(s)\right)+\frac{C(t)-C(0)}{(\omega+\mu) t}+\frac{A(t)-A(0)}{(\alpha+\mu+d) t}\right\} .
\end{aligned}
$$

If $R_{3}>1$, then

$$
\begin{aligned}
\liminf _{t \rightarrow+\infty}\langle I(t)\rangle & \geq \frac{\lambda(\omega+\mu)(\alpha+\mu+d)\left(\mu+\gamma+\rho+\frac{\sigma^{2}}{2}\right)}{\beta(\mu+d)[(\omega+\mu)(\alpha+\mu+d+\rho)+\gamma(\alpha+\mu+d)]}\left(R_{3}-1\right) \\
& >0 \quad \text { almost surely. }
\end{aligned}
$$

\section{Numerical simulations}

In this section, we use Milstein's method [18] to simulate stochastic model (1.2) with a numerical scheme for stochastic model (1.2) given by

$$
\left\{\begin{aligned}
S_{k+1}= & S_{k}+\left[\lambda-\mu S_{k}-\frac{\beta S_{k} I_{k}}{S_{k}+I_{k}+C_{k}+A_{k}}\right] \Delta t \\
& -\frac{\sigma S_{k} I_{k}}{S_{k}+I_{k}+C_{k}+A_{k}} \sqrt{\Delta t} \xi_{k}-\frac{\sigma^{2}}{2}\left(\frac{S_{k} I_{k}}{S_{k}+I_{k}+C_{k}+A_{k}}\right)^{2}\left(\xi_{k}^{2}-1\right) \Delta t, \\
I_{k+1}= & I_{k}+\left[\frac{\beta S_{k} I_{k}}{S_{k}+I_{k}+C_{k}+A_{k}}-(\mu+\gamma+\rho) I_{k}+\alpha A_{k}+\omega C_{k}\right] \Delta t \\
& +\frac{\sigma S_{k} I_{k}}{S_{k}+I_{k}+C_{k}+A_{k}} \sqrt{\Delta t} \xi_{k}+\frac{\sigma^{2}}{2}\left(\frac{S_{k} I_{k}}{S_{k}+I_{k}+C_{k}+A_{k}}\right)^{2}\left(\xi_{k}^{2}-1\right) \Delta t, \\
C_{k+1}= & C_{k}+\left[\gamma I_{k}-(\mu+\omega) C_{k}\right] \Delta t, \\
A_{k+1}= & A_{k}+\left[\rho I_{k}-(\alpha+\mu+d) A_{k}\right] \Delta t,
\end{aligned}\right.
$$

where $\xi_{k}, k=1,2, \ldots, n$, are independent Gaussian random variables $N(0,1)$. 
Table 1 Parameters of the HIV/AIDS model (1.2)

\begin{tabular}{llll}
\hline Symbol & Description & Value & References \\
\hline$\lambda$ & Recruitment rate & 10,724 & {$[19]$} \\
$\mu$ & Natural death rate & 0.3 & Assumed \\
$\beta$ & HIV transmission rate & 0.5 & Assumed \\
$\gamma$ & HIV treatment rate for I & {$[0.01,0.05,0.07]$} & Assumed \\
$\rho$ & Default treatment rate for I & 0.1 & {$[20]$} \\
$\alpha$ & AlDS treatment rate & 0.33 & {$[21]$} \\
$\omega$ & Default treatment rate for C & {$[0.9,0.8,0.1]$} & Assumed \\
$d$ & AlDS induced death rate & 1 & {$[22]$} \\
\hline
\end{tabular}

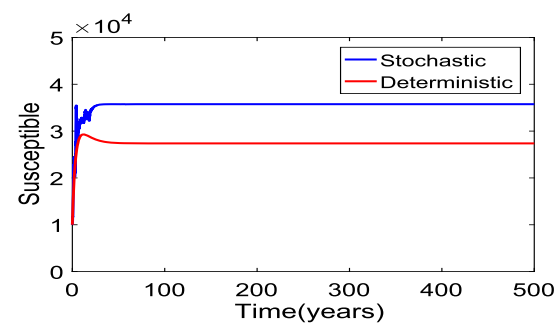

(a)

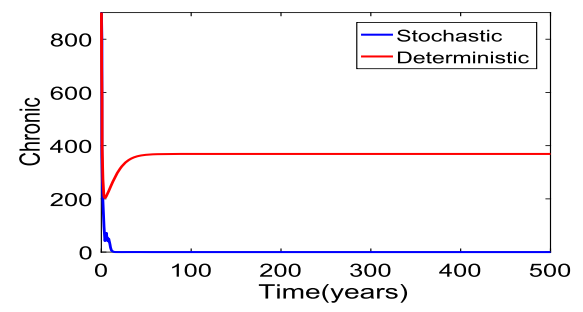

(c)

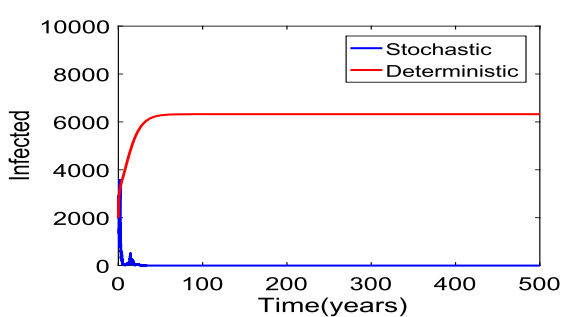

(b)

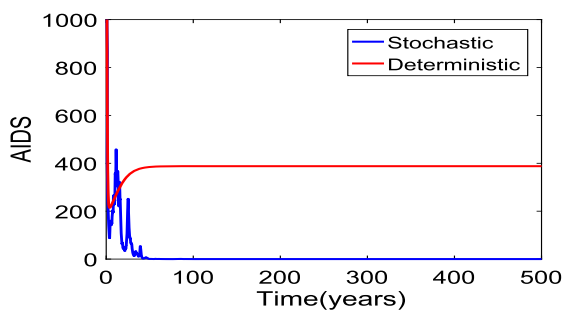

(d)

Figure 1 The path $S(t), I(t), C(t)$, and $A(t)$ for models (1.1) and (1.2), when $R_{1}=0.651<1$ and $R_{0}=1.259>1$

Firstly, we choose $\sigma=0.8, \gamma=0.07, \omega=0.9$, and other parameter values given by Table 1 . In this case, we have

$$
\begin{aligned}
& R_{1}=\frac{\beta^{2}}{2 \sigma^{2} \mu}=0.651<1, \\
& R_{2}=\frac{\beta}{\left(\mu+\frac{\sigma^{2}}{2}\right)}=0.806<1, \quad \sigma^{2}-\beta=0.14>0, \\
& R_{3}=\frac{\beta}{\mu+\gamma+\rho+\frac{\sigma^{2}}{2}}=0.633<1,
\end{aligned}
$$

then the disease $I(t)$ will die out (see Theorem 3.1 and Fig. 1(b)). In addition, the basic reproduction number of the corresponding deterministic model $R_{0}=1.259>1$, this means that the corresponding deterministic model (1.1) has an endemic equilibrium which is globally asymptotically stable, as shown in Fig. 1(a)-(d). 
Secondly, we choose $\sigma=0.645, \gamma=0.05, \omega=0.8$, and other parameter values given by Table 1. In this case, we have

$$
\begin{aligned}
& R_{1}=\frac{\beta^{2}}{2 \sigma^{2} \mu}=1.002>1, \\
& R_{2}=\frac{\beta}{\left(\mu+\frac{\sigma^{2}}{2}\right)}=0.9842<1, \quad \sigma^{2}-\beta=-0.084<0, \\
& R_{3}=\frac{\beta}{\mu+\gamma+\rho+\frac{\sigma^{2}}{2}}=0.7599<1,
\end{aligned}
$$

then the disease $I(t)$ will die out (see Theorem 3.1 and Fig. 2(b)). In addition, the basic reproduction number of the corresponding deterministic model $R_{0}=1.271>1$, this means that the corresponding deterministic model (1.1) also has an endemic equilibrium which is globally asymptotically stable, as shown in Fig. 2. Furthermore, we choose $\gamma=0.07$, $\omega=0.9$, and $\sigma=0.8,1,1.2,1.4$, and other parameter values given by Table 1 to study the impact of $\sigma$ on the dynamics for the SDE SICA model (1.2). In this case, we can obtain the values given in Table 2. Theorem 3.1 reveals the numerical results shown in Fig. 3.

Our results reveal that random perturbations in the environment can restrain the spread of the disease (see Fig. 2), and the bigger the intensity of the random perturbation, the

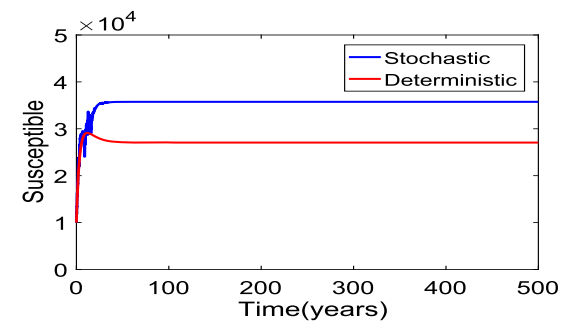

(a)

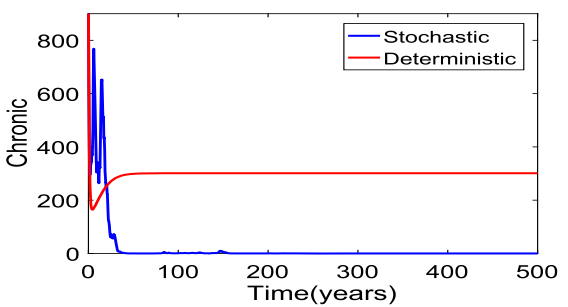

(c)

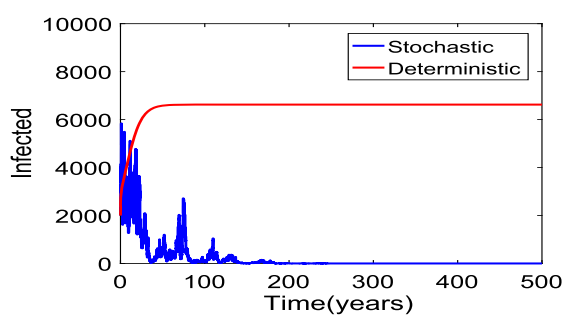

(b)

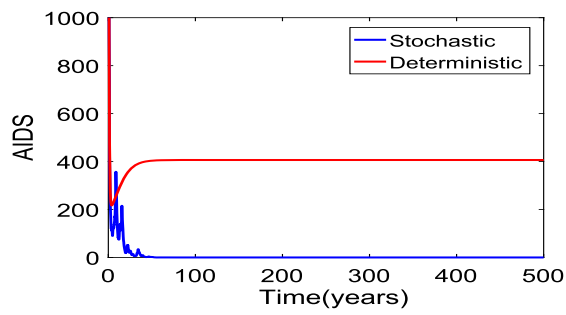

(d)

Figure 2 The path $S(t), I(t), C(t)$, and $A(t)$ for model (1.1) and (1.2), when $R_{2}=0.9842<1, \sigma^{2}-\beta=-0.084<0$, and $R_{0}=1.271>1$

Table 2 The values with $\sigma=0.8,1,1.2,1.4$

\begin{tabular}{lllll}
\hline$\sigma$ & 0.8 & 1 & 1.2 & 1.4 \\
\hline$R_{1}$ & 0.4156 & 0.266 & 0.1847 & 0.1357 \\
$R_{2}$ & 0.6329 & 0.5155 & 0.4202 & 0.3448 \\
$\sigma^{2}-\beta$ & 0.14 & 0.5 & 0.94 & 1.46 \\
\hline
\end{tabular}




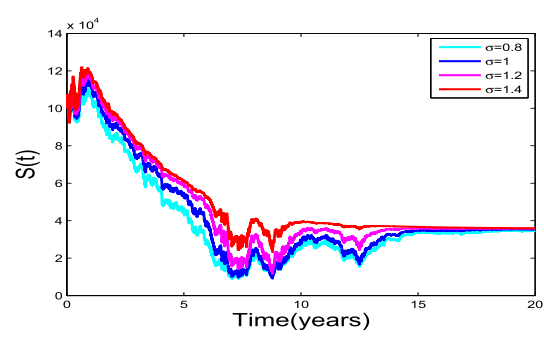

(a)

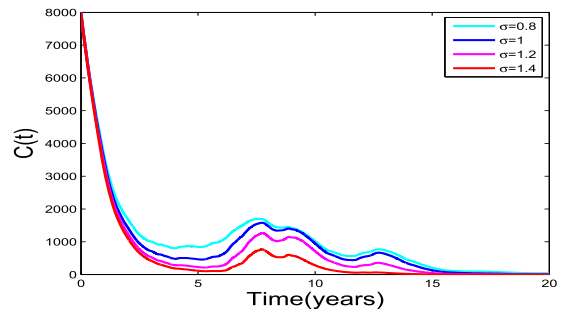

(c)

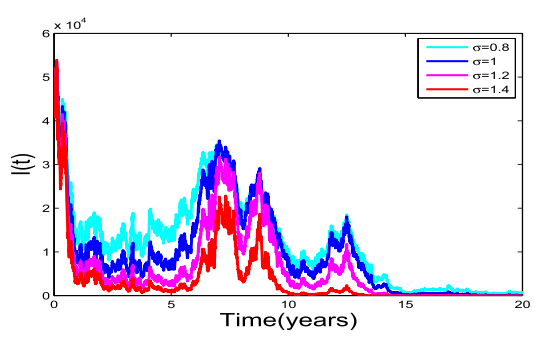

(b)

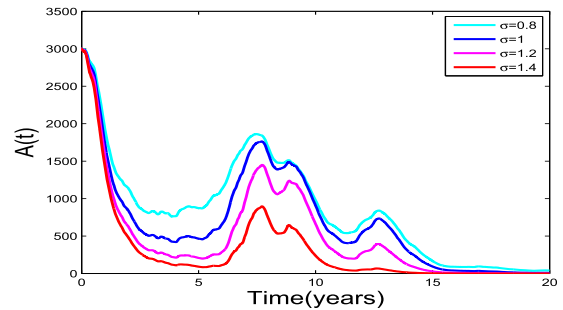

(d)

Figure 3 The path of $(S(t), /(t), C(t), A(t))$ for stochastic model (1.2) when $\sigma=0.8,1,1.2,1.4$

Table 3 The values with $\beta=0.2,0.4,0.6,0.8,1,1.2$

\begin{tabular}{llllccc}
\hline$\beta$ & 0.2 & 0.4 & 0.6 & 0.8 & 1 & 1.2 \\
\hline$R_{1}$ & 0.104 & 0.417 & 0.937 & 1.667 & 2.604 & 3.75 \\
$R_{2}$ & 0.323 & 0.645 & 0.968 & 1.290 & 1.613 & 1.935 \\
$\sigma^{2}-\beta$ & 0.44 & 0.24 & 0.04 & -0.16 & -0.36 & -0.56 \\
$R_{3}$ & 0.253 & 0.506 & 0.759 & 1.013 & 1.266 & 1.519 \\
\hline
\end{tabular}

faster the disease dies out (see Fig. 3). However, deterministic models ignore this, so it is essential to introduce stochastic perturbations into deterministic models.

Thirdly, we fix $\sigma=0.8$ and choose $\beta=0.2,0.4,0.6,0.8,1,1.2$ and other parameters taken as in Table 1 to study the impact of $\beta$ on the dynamics for the SDE SICA model (1.2). In this case, we can obtain the values given in Table 3 , and the numerical results show that the smaller the transmission rate, the faster the disease dies out (see Fig. 4).

Finally, we choose $\sigma=0.2, \gamma=0.01, \omega=0.1$ and other parameter values given by Table 1 . In this case, we have

$$
\begin{aligned}
& R_{1}=\frac{\beta^{2}}{2 \sigma^{2} \mu}=10.417 \\
& R_{2}=\frac{\beta}{\left(\mu+\frac{\sigma^{2}}{2}\right)}=1.563>1, \quad \sigma^{2}-\beta=-0.46<0, \\
& R_{3}=\frac{\beta}{\mu+\gamma+\rho+\frac{\sigma^{2}}{2}}=1.163>1,
\end{aligned}
$$

then the disease $I(t)$ will be persistence in mean, namely, the disease will prevail (see Theorem 3.2 and Fig. 5(b)). 


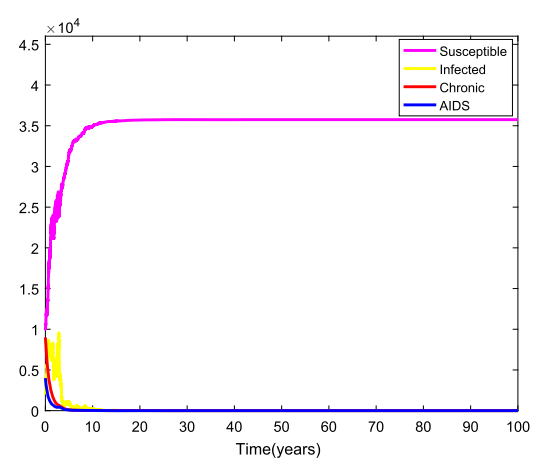

(a) $\beta=0.2$

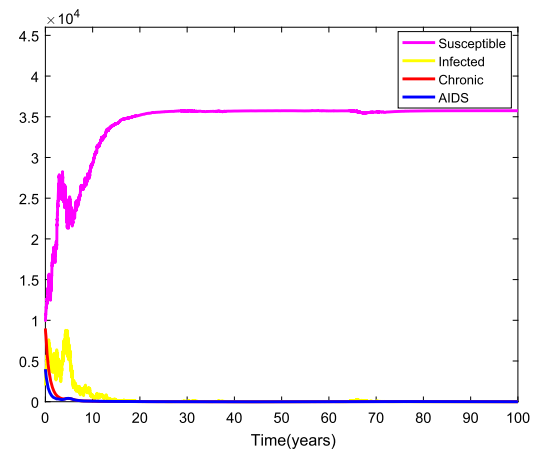

(c) $\beta=0.6$

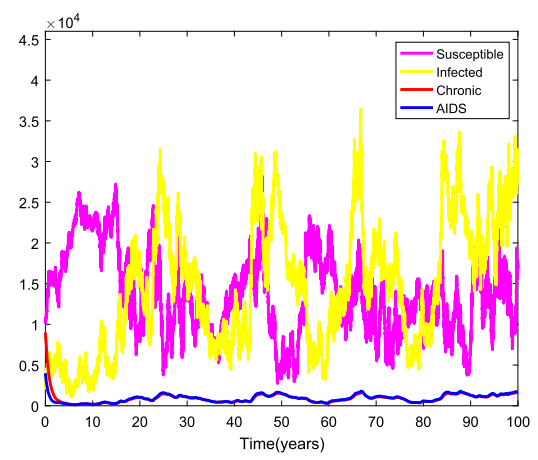

(e) $\beta=1$

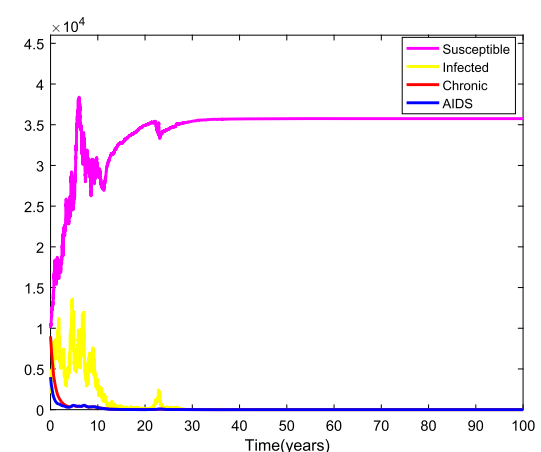

(b) $\beta=0.4$

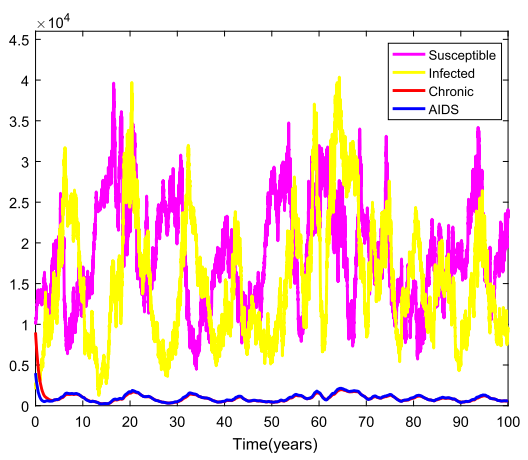

(d) $\beta=0.8$

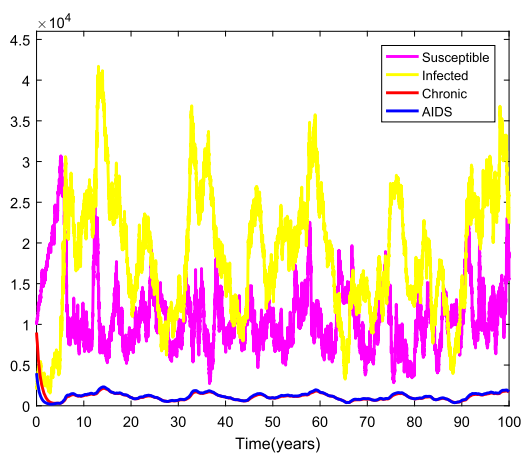

(f) $\beta=1.2$

Figure 4 The path of $(S(t), I(t), C(t), A(t))$ for stochastic model (1.2) when $\sigma=0.8$

By numerical simulation, the results of numerical simulations show that HIV can be controlled by increasing the intensity of interference and reducing the transmission rates shown in Fig. 6 (e.g., increased HIV prevention campaigns, condom use, etc.)

\section{Conclusion}

This paper studied the extinction and persistence of a stochastic SICA epidemic model with standard incidence rate for HIV transmission. Firstly, we analyze that model (1.2) has a unique global positive solution for any initial value. Secondly, by Theorem 3.1, we can find that when $R_{1}=\frac{\beta^{2}}{2 \sigma^{2} \mu}<1$ or $\sigma^{2}<\beta$ and $R_{2}=\frac{\beta}{\left(\mu+\frac{\sigma^{2}}{2}\right)}<1$, disease will die out (see The- 


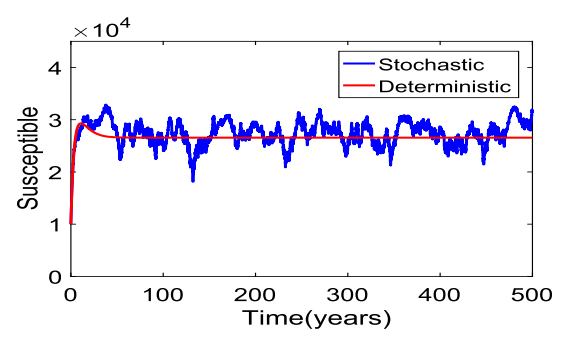

(a)

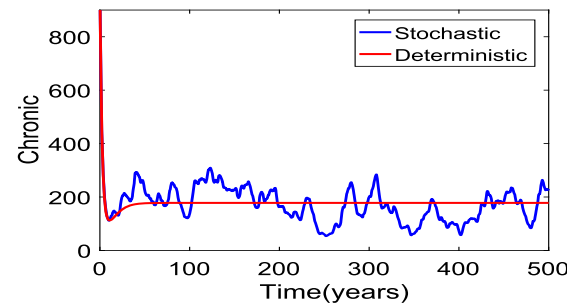

(c)

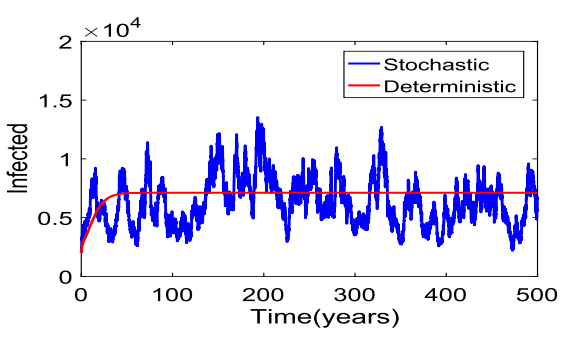

(b)

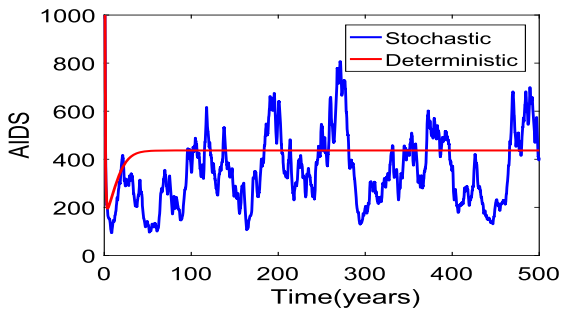

(d)

Figure 5 The path $S(t), I(t), C(t)$, and $A(t)$ for models (1.1) and (1.2), when $R_{3}=1.163>1$ and $R_{0}=1.291>1$

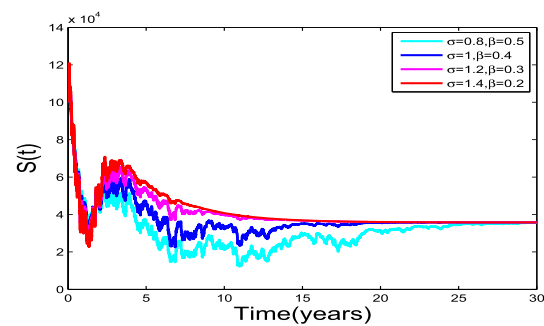

(a)

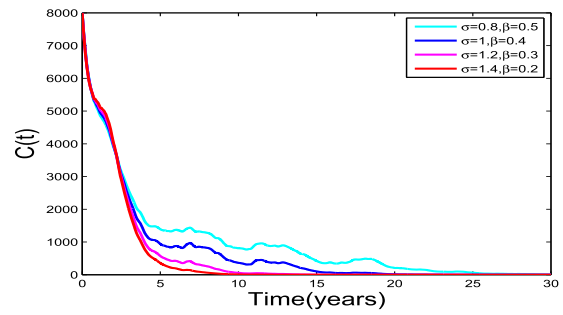

(c)

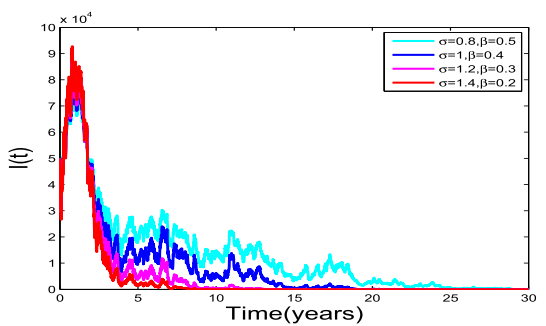

(b)

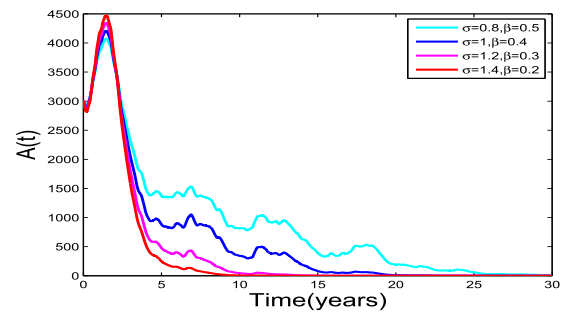

(d)

Figure 6 The path of $(S(t), I(t), C(t), A(t))$ for stochastic model (1.2) when $\sigma=0.8,1,1.2,1.4$ and $\beta=0.5,0.4,0.3,0.2$

orem 3.1 and Fig. 1 and Fig. 2). Furthermore, $\lim _{t \rightarrow+\infty} S(t)=\frac{\lambda}{\mu}$ (see Theorem 3.1), but for the corresponding deterministic model (1.2), $R_{0}>1$, there exists an endemic equilibrium, which means that a stochastic perturbation can suppress the outbreak of the disease (see Fig. 1 and Fig. 2), and the bigger the intensity of the random perturbation, the faster the disease dies out (see Fig. 3). However, deterministic models do not take this into account, so it is essential to include a stochastic element in deterministic models. In addition, we fix 
$\sigma$ to study the impact of $\beta$ on the dynamics for the SDE SICA model (1.2). In this case, we can find that the greater the rate of transmission, the higher the number of people infected (see Fig. 4).

Finally, if $R_{3}=\frac{\beta}{\mu+\gamma+\rho+\frac{\sigma^{2}}{2}}>1$, the disease will be persistence in mean, namely, the disease will prevail (see Theorem 3.2 and Fig. 5).

Through numerical simulations, we can conclude that it is possible to reduce the transmission coefficient of HIV while increasing the strength of the stochastic perturbation to reduce the risk of HIV transmission, the simulation results are shown in Fig. 6.

On the other hand, in this paper, we only consider the effect of random perturbations on HIV transmission rate $\beta$, we can also study the effect of random perturbations on another parameter such as natural death rate, HIV treatment rate, AIDS induced death rate, and so on. In addition, in this paper, we only consider the effect of white noise. In fact, there are some random perturbations which cannot be modeled by white noises, for example, the telephone and Lévy noise, see [23-26] and the references therein. On the other hand, there have also been extensive numerical works to establish the positive property of numerical solutions for certain physical models (see [27-29]). We leave these investigations for future work.

\section{Acknowledgements}

The authors would like to thank the editor and the anonymous reviewers for their valuable comments and constructive suggestions.

\section{Funding}

This research was supported by Program for Tianshan Innovative Research Team of Xinjiang Uygur Autonomous Region China (2020D14020).

\section{Availability of data and materials}

Data sharing not applicable to this article as no datasets were generated or analysed during the current study.

\section{Competing interests}

The authors declare that they have no competing interests.

\section{Authors' contributions}

All authors contributed equally and significantly in writing this paper. All authors read and approved the final manuscript.

\section{Publisher's Note}

Springer Nature remains neutral with regard to jurisdictional claims in published maps and institutional affiliations.

Received: 27 July 2020 Accepted: 21 April 2021 Published online: 19 May 2021

\section{References}

1. Silva, C.J., Torres, D.F.M.: A TB-HIV/AIDS coinfection model and optimal control treatment. Discrete Contin. Dyn. Syst. 35(9), 1-25 (2015)

2. Naik, P.A., Yavuz, M., Zu, J.: The role of prostitution on HIV transmission with memory: a modeling approach, AEJ. Alex. Eng. J. 59(4), 2513-2531 (2020)

3. Naik, P.A., Zu, J., Owolabi, K.: Global dynamics of a fractional order model for the transmission of HIV epidemic with optimal control. Chaos Solitons Fractals 137, 1-30 (2020)

4. Silva, C.J., Torres, D.F.: A SICA compartmental model in epidemiology with application to HIV/AIDS in Cape Verde. Ecol. Complex. 30, 70-75 (2017)

5. May, R.: Stability and Complexity in Model Ecosystems, Princetom. Princeton University Press, Princeton (1973)

6. Gray, A., Greenhalgh, D., Hu, L., Mao, X., Pan, J.: A stochastic differential equation SIS epidemic model. SIAM J. Appl. Math. 71(3), 876-902 (2011)

7. Zhang, X.B., Huo, H.F., Xiang, H., Shi, Q., Li, D.: The threshold of a stochastic SIQS epidemic model. Phys. A, Stat. Mech. Appl. 482, 362-374 (2017)

8. Zhang, X.B., Huo, H.F., Xiang, H., Meng, X.Y.: Dynamics of the deterministic and stochastic SIQS epidemic model with non-linear incidence. Appl. Math. Comput. 243, 546-558 (2014)

9. Zaman, G., Han, K.Y., Jung, I.H.: Stability and optimal vaccination of an SIR epidemic model. Biosystems 93(3), 240-249 (2008)

10. Zhao, Y., Jiang, D.: Dynamics of stochastically perturbed SIS epidemic model with vaccination. Abstr. Appl. Anal. 2013, $517439(2013)$ 
11. Liu, Q., Chen, Q.: Analysis of the deterministic and stochastic SIRS epidemic models with nonlinear incidence. Phys. A, Stat. Mech. Appl. 428, 140-153 (2015)

12. Jasmina, D., Silva, C.J., Torres, D.F.M.: A stochastic SICA epidemic model for HIV transmission. Appl. Math. Lett. 84 168-175 (2018)

13. Wang, C., Agarwal, R.P., Rathinasamy, S.: Almost periodic oscillations for delay impulsive stochastic Nicholson's blowflies timescale model. Comput. Appl. Math. 37(3), 3005-3026 (2018)

14. Rathinasamy, S., Ramalingam, S., Boomipalagan, K., Wang, C., Ma, Y.K.: Finite-time nonfragile synchronization of stochastic complex dynamical networks with semi-Markov switching outer coupling. Complexity 2018, 1-13 (2018)

15. Wang, C., Agarwal, R.P.: Almost periodic solution for a new type of neutral impulsive stochastic Lasota-Cwazewska timescale model. Appl. Math. Lett. 70, 58-65 (2017)

16. Wang, C.: Existence and exponential stability of piecewise mean-square almost periodic solutions for impulsive stochastic Nicholson's blowflies model on time scales. Appl. Math. Comput. 248, 102-112 (2014)

17. Mao, X.: Stochastic Differential Equations and Their Applications. Horwood, Chichester (1997)

18. Higham, D.J.: An algorithmic introduction to numerical simulations of stochastic differential equations. SIAM Rev. 43, 525-546 (2001)

19. World Bank Data: Cabo Verde, World Development Indicators (2014)

20. Song, B., Gumel, A., Podder, C.N., Sharomi, O.: Mathematical analysis of the transmission dynamics of HIV/TB coinfection in the presence of treatment. Math. Biosci. Eng. 5(1), 145-174 (2008)

21. Watmough, J., Musgrave, J.: Examination of a simple model of condom usage and individual withdrawal for the HIV epidemic. Math. Biosci. Eng. 6(2), 363-376 (2009)

22. Marcel, Z., Matthias, E.: Progression and mortality of untreated HIV-positive individuals living in resource-limited settings: update of literature review and evidence synthesis. Report on UNAIDS obligation no HQ/05/422204,(2006)

23. Liu, M., Bai, C.: Optimal harvesting of a stochastic mutualism model with Levy jumps. Appl. Math. Comput. 276 301-309 (2016)

24. Zhou, Y., Zhang, W.: Threshold of a stochastic SIR epidemic model with Levy jumps. Phys. A, Stat. Mech. Appl. 446 204-216 (2015)

25. Chen, C., Kang, Y:: Dynamics of a stochastic multi-strain SIS epidemic model driven by Levy noise. Commun Nonlinear Sci. Numer. Simul. 42, 379-395 (2017)

26. Zhang, X.B., Shi, Q., Ma, S., Huo, H., Li, D.: Dynamic behavior of a stochastic SIQS epidemic model with Levy jumps. Nonlinear Dyn. 93, 1481-1493 (2017)

27. Liu, J.G., Wang, C.: Positivity property of second-order flux-splitting schemes for the compressible Euler equations. Discrete Contin. Dyn. Syst., Ser. B 3(2), 201-228 (2012)

28. Chen, W., Wang, C., Wang, X., Wise, S.M.: Positivity-preserving, energy stable numerical schemes for the Cahn-Hilliard equation with logarithmic potential. J. Comput. Phys. X 3, 100031 (2019)

29. Dong, L., Wang, C., Zhang, H., Zhang, Z.: A positivity-preserving second-order BDF scheme for the Cahn-Hilliard equation with variable interfacial parameters. Commun. Comput. Phys. 28(3), 967-998 (2020)

\section{Submit your manuscript to a SpringerOpen ${ }^{\circ}$ journal and benefit from:}

- Convenient online submission

- Rigorous peer review

- Open access: articles freely available online

- High visibility within the field

- Retaining the copyright to your article

Submit your next manuscript at $\gg$ springeropen.com 